\title{
Toucher au corps ${ }^{1}$
}

\section{Linda Bonin}

This article offers a reading of Black Words, a collaborative work by Denise Desautels and Betty Goodwin. Through the gesture of drawing which unites the writing practice of the one and the visual art of the other, this book reimagines writing as an experience of touch. The singularity of a stroke or line becomes a touch whose import is no longer a sign to be deciphered but the trace of a life in which the body presents itself as a site of memory and passage.

Il n'arrive donc rien d'autre à l'écriture, s'il lui arrive quelque chose, que de toucher.

- Jean-Luc Nancy

Selon Jean-Luc Nancy, l'écriture privilégie, plus que tout autre sens, le toucher puisqu'elle fait « du sens une touche » (Corpus 13). Si toucher, c'est « entrer en contact, avec mouvement » ou « arriver à rencontrer quelqu'un par un intermédiaire » ou encore « affecter», ces caractéristiques peuvent tout aussi bien définir l'expérience de l'écriture. L'« expérience dont l'origine dit quelque chose de la traversée ; mais d'une traversée avec le corps, d'un espace qui n'est pas donné d'avance mais qui s'ouvre à mesure qu'on avance » (Derrida, Points de suspension 221) dit également un certain passage des frontières, de ce que conventionnellement on pense être des limites, par exemple celles qui existeraient entre l'écriture et l'art, plus spécifiquement entre la poésie et le dessin. Aussi, par écriture, faut-il entendre le geste de tracer, $d^{\prime}$ ' ouvrir plus ou moins un chemin [un espace] en faisant une trace ", voire "former en faisant plusieurs traits ", c'est-à-dire « dessiner » et " former par les traits de l'écriture », voire " écrire » la singularité d'un trait, d'une touche, qui soit celle d'une main, d'un corps. C'est pourquoi « toucher au corps » s'annonce à l'infinitif pour signifier qu'il ne s'agit pas d'exposer une subjectivité par laquelle se constitue un sujet écrivant, mais tout au contraire une certaine dépossession, un certain aveuglement qui 
paradoxalement donne à voir la vérité des corps, leur existence, car c'est bien ce que révèle Black Words de Denise Desautels et Betty Goodwin.

Là où « c'est de plus en plus souvent la nuit » $(B W)$, les mots ne sont plus des signes mais les traces d'une vie « tenue à distance » $(B W)$, d'une vie qui " s'épuise lentement" $(B W)$. Le choix même du titre, Black Words, annonce d'emblée l'obscurité d'où jaillissent les mots et l'incertitude quant à leur pouvoir de communication : " les mots n'atteindront jamais l'autre » $(B W)$. Si les mots ne peuvent à eux seuls atteindre l'autre, peut-être que la bouche parviendra à ouvrir un espace où il sera possible d'effleurer l'autre, de s'effleurer comme autre :

je me demande ce qui s'ouvre
une bouche peut-être
tendue vers d'autres bouches
qu'elle épie qu'elle tourmente qu'elle avale parfois
avoir faim avoir soif
le tableau est désert
le siècle se referme sans visage
continuer à vivre en sursis échappe aux sens (BW)

Sans visage, la bouche se fait lieu de passage pour ce qui demeure sans figure et sans nom. Dans ce mouvement d'ouvert/fermé - puisque suite à ce qui s'ouvre "le siècle se referme ", comme si devant l'espace (l'ouvert), le temps s'effaçait, s'éclipsait de la scène - il ne reste que l'étendue d'une pensée, d'un je qui s'affecte et se voit dépossédé de lui-même : " continuer à vivre en sursis échappe aux sens ». L'infinitif de ce dernier vers dit bien « qu'une certaine position égologique ou subjectivante se défait et ne peut plus tenir » (Derrida, Soussana, Nouss 22), que le corps échappe à l'entendement d'une logique raisonnante et linéaire où même le temps, dans sa linéarité classique (passé, présent, futur), ne peut que s'éclipser, car " vivre en sursis ", c'est vivre en différé, comme si le présent vivant était toujours déjà passé, toujours déjà empreint de son double qu'est la mort : « je ne sens presque plus le reste de mon corps / on dirait un corridor de plomb / sans commencement ni fin / la mort rejoint tous les paysages » $(B W)$. L'extrait d'Ingeborg Bachmann en exergue du texte met aussi ce fait en évidence : "C'est à présent que ce jour / devient une énigme, un jour vide, / un jour volé où j'ai vieilli, / où je ne me suis pas défendue, / où j'ai laissé faire je ne sais quoi. ». 
Plus d'une fois Desautels a recours à l'infinitif, plus loin elle écrit : " avancer revenir piétiner / mes pas décomposent minutieusement l'oubli » $(B W)$. Présentés sans ponctuation, les trois verbes du premier vers, introduisent, d'un même souffle, trois actions différentes qui semblent constituer un seul et même mouvement, comme si « avancer » impliquait qu'il faille revenir, était d'avance empreint de son contraire, de son double, et ne pouvait dès lors qu'évacuer le sujet de l'action. Toutefois, cette agitation n'est pas vaine puisque des " pas décomposent minutieusement l'oubli », ceux d'un corps qui se souvient avoir oublié, avoir « laissé faire je ne sais quoi ». Et quel est donc ce « je ne sais quoi » sinon l'oubli de la vie, $\mathrm{du}$ vivant, qu'un mouvement même de sur place (le piétinement) vient éveiller signalant ainsi que l'infigurable n'est nul autre que soi, corps et pensée : " une pensée inclinée vers le monde / laisse des traces / écrire est un paysage sonore / l'oeil la bouche l'épaule la main / lient les mots et les choses » $(B W)$ - les « lient », c'est-à-dire à la fois les lire et les lier, les lire d'une toute autre manière ces mots puisqu'en les liant aux choses ils ne sont plus des signes à déchiffrer mais les traces d'une vie où le corps apparaît dans toute son étrangeté, sa vulnérabilité, sa douleur, sa force, son poids. Il en va de même pour les dessins de Betty Goodwin avec lesquels le texte de Desautels entretient une véritable relation. Toujours présent dans l'oeuvre de Goodwin, le corps laisse souvent entendre " que la subjectivité ne peut pas tenir son centre contre les forces de désintégration qui l'entourent. (On pense à ses dessins dans lesquels les têtes sont arrachées aux corps ou expulsent leur contenu comme si elles éclataient) » (Bradley 52). De même, dans Black Words, les corps sont davantage le support d'un affect que d'un percept - souffrants, aliénés, parfois matières propulsées ou attirées par on ne sait quelle force obscure. Épuisés d'être objet de représentation, ils ne se donnent point à lire en tant que portrait, icône ou signe. Asexués - on ne peut distinguer s'il s'agit d'un homme ou d'une femme -, sans ego, sans identité autre qu'humaine, ces corps sont dilués, vaporeux, évanescents. Ils se fondent au papier (au support), au monde, ils se montrent en s'effaçant et deviennent lieu de passage et de mémoire, mais d'une mémoire si lointaine qu'elle se nomme oubli.

Cette mémoire, depuis longtemps, Desautels l'explique dans son propre travail par la figure de l'archéologue, signalant ainsi la traversée des différentes strates de la mémoire, d'un forage où l'écriture est susceptible de percer l'oubli. Cette pratique donne à penser que ce qui est à l'oeuvre dans ce travail de fouilles est en fait un tracé de son être corps au monde. 
Tout comme le trait des dessins de Goodwin, l'archéologue n'est pas sans rappeler les grottes de Lascaux où la peinture « est d'abord la monstration du commencement de l'être » (Nancy, Les Muses 122). Et ce qu'interrogent l'écrivaine et l'artiste dans Black Words, c'est bien le sens de l'être au monde où le corps évoque le passage du visible à l'invisible, d'un visible qui naît de l'aveuglement, d'une vision qui relève davantage de la mémoire que de la perception.

Alors, l'oeil qui jusque-là n'avait fait que percevoir les choses se découvre voyant. [...] Et c'est toujours aussi y voir dans la nuit de la grotte, le regard droit tendu dans la profondeur noire. Et il y voit l'Idée, l'étrangère, la figure : il est ouvert par elle et en elle, il est rythmé sur elle, et c'est elle, le Monstre qu'il est lui-même. Il voit l'invisible, et l'évanouissement de sens de sa propre présence au monde. (Nancy, Les Muses 132)

C'est pourquoi, dans l'oeuvre de Goodwin et de Desautels, l'être est trace, reste de signes, vestiges du vivant et sa mémoire, un tombeau d'instants morts : " "Black Words" disait la voix / excuse de l'odeur / cendre et peau jusqu'à satiété / mon corps a sa propre bonté / et remue bêtement comme une chose vivante $"(B W)$.

La cendre, tout comme la bouche, le corps, le pas et le " corridor de plomb » évoquent le thème du passage si caractéristique du travail de Goodwin dans lequel il fait référence non seulement au temps et à la présence physique, mais aussi à la difficulté de communiquer (Bradley 49). Le passage lui-même n'est pas sans correspondre à l'idée de trace, de vestige, puis de cendre :

la cendre, c'est évidemment une trace - en général on pense, comme première figure de la trace à celle du pas, dans le chemin, dans l'acheminement, du pas qui laisse une empreinte, une trace ou un vestige -; mais « cendre » dit mieux ce que je voulais dire sous le nom de trace à savoir quelque chose qui reste sans rester. Qui n'est ni présent ni absent ; qui se détruit lui-même, soi-même, qui se consume totalement, qui est un reste sans reste. C'est-à-dire quelque chose qui n'est pas. [...] La cendre n'est pas, cela signifie qu'elle témoigne sans témoigner. Elle témoigne de la disparition du témoin, si on peut dire. Elle témoigne de la disparition de la mémoire. (Derrida, Points de suspension 222) 
C'est peut-être là la bonté dont parle Desautels, celle du corps qui, entre être et non être, demeure une ouverture qui ne cesse de s'ouvrir, un passage " sans commencement ni fin » entre vie et mort, mémoire et oubli, présence et absence, transparence et opacité, inscription et effacement. Et si ce corps " remue bêtement comme une chose vivante " et non comme une chose morte, $c^{\prime}$ est que ce à quoi il touche n'a plus rien à voir avec l'ordre de la supposition (d'un sens donné), mais avec " celui de la venue » (Nancy, Corpus 83) : « je suis absorbée par des intentions de lumière / par la vie comme un geste dressé / rouge vif dans le poème " $(B W)$. Le rouge, symbole de la douleur, de la souffrance et de la vie est aussi très présent dans les dessins de Goodwin. Cette couleur, appliquée sur un support (papier géofilm), qui, par sa translucidité, son grain et sa texture, s'apparente à celle d'une peau, rappelle le corps, la chair, le sang. Alors, si ce " geste dressé / rouge vif dans le poème " signifie la vie qui s'élève, s'érige dans l'oeuvre, il signifie tout autant la douleur d'une vie domptée, domestiquée, assujettie à un ordre. Ce double sens n'est pas sans rappeler Le théâtre et son double d'Antonin Artaud auquel se sont intéressées l'artiste et l'écrivaine. Le théâtre de la cruauté ne visait-il pas à « traverser et restaurer de part en part l'"existence" et la "chair" » (Derrida, L'écriture et la différénce 341) ? N'était-il pas " la vie elle-même en ce qu'elle a d'irreprésentable " (343) ? La cruauté, rigoureuse nécessité de "toucher au corps ", prend forme dans Black Words par l'expérience d'une écriture qui éveille la mémoire émotive et sensorielle $d^{\prime} u n$ corps, autrement dit d'une existence, qui en se traversant atteint l'autre, cet invisible, toujours menacé de disparition.

\section{Notes}

${ }^{1}$ Ce texte s'inscrit dans le cadre d'une recherche subventionnée par la Fondation UQÀM, le Fonds pour la Formation de Chercheurs et l'Aide à la Recherche, le Conseil de Recherche en Sciences Humaines du Canada et le programme de perfectionnement UQÀM-SCCUQ. 


\section{Oeuvres cités}

Bradley, Jessica. Betty Goodwin. Signs of life - Signes de vie. Art Gallery of Windsor et Musée des beaux-arts du Canada, 1995-1996.

Derrida, Jacques. L'écriture et la différence. Paris : Seuil, 1967.

- Points de suspension. Entretiens. [choisis et présentés par Elisabeth Weber]. Paris : Galilée, 1992.

Derrida, Jacques, Gad Soussana, et Alexis Nouss. Dire l'événement, est-ce possible? Séminaire de Montréal, pour Jacques Derrida. Montréal : L'Harmattan, 2001.

Desautels, Denise. Black Words [Impressions laser et dessins originaux de Betty Goodwin]. Imprimé à Montréal et édité à Paris par « Collectif Génération », 1990 [n.p.].

Nancy, Jean-Luc. Corpus. Paris : Métailié, 1992.

—. Les Muses. Paris : Galilée, 1994. 